\title{
Die Erneuerung beruflicher Lern- und Karrierewege am Beispiel der Industriekaufleute
}

Welchen Stellenwert hat die Facharbeit für die Beschäftigtengruppe der Industrieangestellten? Diese Frage gewinnt für die Zukunft der Facharbeit zunehmend an Bedeutung. Facharbeit findet faktisch immer weniger in der unmittelbaren Produktion und immer mehr in den planenden, verwaltenden, entwickelnden oder kaufmännischen Bereichen der Unternehmen statt. Der Beitrag beschreibt am Beispiel der Berufsgruppe der Industriekaufleute zum einen die Anforderungen, die an Industrieangestellte gestellt werden. Zum anderen werden die Veränderungen analysiert, die sich mit Blick auf ihre Lern- und Karrierepfade in den letzten Jahren vollzogen haben.

TABEA BROMBERG, THOMAS HAIPETER, ANGELIKA KÜMMERLING

\section{Industriekaufleute und berufliche Fachlichkeit}

Die Industriekaufleute sind eine der größten Gruppen unter den kaufmännischen Berufen. Zusammen mit den Bürokaufleuten und anderen kaufmännischen Berufsgruppen wie den Automobil- oder den Logistikkaufleuten bilden sie die Beschäftigtengruppe der kaufmännischen Angestellten in den Industriebranchen. Doch anders als die Bürokaufleute, deren Qualifikationsprofil vor allem auf verwaltende Tätigkeiten ausgerichtet ist und die auch in Dienstleistungsunternehmen ausgebildet und eingesetzt werden, haben die Industriekaufleute ein sektorspezifisches Qualifikationsprofil. Sie können mithin als der Prototyp kaufmännischer Angestellter in der Industrie betrachtet werden.

In den letzten Jahren haben sich die organisatorischen und inhaltlichen Rahmenbedingungen der Arbeit kaufmännischer Angestellter in Industrieunternehmen grundlegend verändert. Um Mindestrenditen zu erreichen, bauen die Unternehmen Beschäftigung ab und machen Organisationseinheiten und Beschäftigten Leistungs- und Erfolgsvorgaben (Dörre 2012). Standorte werden unter Verlagerungsdruck gesetzt und Beschäftigung wird auch in den Angestelltenbereichen infrage gestellt (Boes/Kämpf 2010). Und schließlich werden Hierarchieebenen abgebaut und operativ verantwortliche Organisationseinheiten geschaffen, die im internen und externen Wettbewerb stehen und gefordert sind, Renditevorgaben unter offenem oder latentem Auslagerungsdruck zu erfüllen. Diese marktzentrierten Kontrollformen erhöhen den Druck auf die Beschäftigten, führen zu höheren Leistungsanforderungen und fördern die Entgrenzung der Arbeitszeiten. Zugleich aber sind in den Angestelltenbereichen der Industrie wachsende Autonomiespielräume und steigende Verantwortung (Kratzer/Nies 2009) ebenso zu beobachten wie neue Anforderungen hinsichtlich der Fremdsprachenkenntnisse oder der interkulturellen Kompetenzen (von Behr 2006). Auch wächst die Bedeutung der Kundenorientierung (Holtgrewe/Voswinkel 2002) und der Interaktionsarbeit mit den Kunden (Dunkel/Rieder 2004). Und nicht zuletzt wird die Arbeit informatisiert (Boes/ Pfeiffer 2006). Damit erhalten soziale Kompetenzen sowie IT- und Softwarekenntnisse eine zunehmende Bedeutung als Anforderungsmerkmale der Angestelltenarbeit.

Welche Folgen haben diese Entwicklungen für die Berufsfachlichkeit und Entwicklungschancen der Industriekaufleute? Anhand der aktuellen Debatten um berufliche Qualifikationen und ihren Stellenwert lassen sich drei Dimensionen der Berufsfachlichkeit unterscheiden, anhand derer die Entwicklung dieser Berufsgruppe zu untersuchen ist. Die erste Dimension betrifft den Stellenwert der dualen Erstausbildung. Dieser ist seit den 1990er Jahren umstritten. Hinweisen auf Funktionsdefizite des dualen Ausbildungssystems (Kern/Sabel 1994; Herrigel/Sabel 1999) und neue Anforderungen wissensbasierter und kundenorientierter Produktion (Baethge 2001; Baethge/Baethge-Kinsky 1998) stehen als Ge- 
genargumente die Bedeutung des Berufsprinzips in neuen Formen der Arbeitsorganisation (Finegold/Wagner 1999) oder die Anpassungsfähigkeit der Berufsbilder (Bosch 2000) gegenüber. Die zweite Dimension bezieht sich auf den Wandel der Lernformen und der Kompetenzentwicklung in der Weiterbildung im Betrieb. Zwischen den Lernformen zeichnet sich eine Gewichtsverschiebung zugunsten unternehmensinterner Angebote und informeller Lernformen ab (Baethge et al. 2006). Die dritte Dimension schließlich betrifft die Bedeutung beruflicher Ausbildungen für die internen Karrierepfade in den Unternehmen. Durch Dezentralisierung und Hierarchieabbau wurden funktionale Organisationsgrenzen abgebaut, die Zahl der Aufstiegspositionen verringert und das Anforderungsprofil zugunsten unternehmerischer Kompetenzen gewandelt (Faust et al. 2000). Damit wurden die alten Aufstiegsmuster fachlicher Kaminkarrieren (Lane 1989) für Angestellte verengt. Zugleich wurden der Ergebnisdruck auf die mittleren Hierarchieebenen verschärft und bisherige Leistungskompromisse aufgekündigt (Kotthoff 1997). Schließlich droht als Folge der Neuausrichtung der Hochschulen und der Einführung des Bachelors sowie generell des Anstiegs der Akademikerquote eine verstärkte Konkurrenz zwischen Beschäftigten mit akademischen Abschlüssen und solchen mit Qualifikationen des beruflichen Ausbildungssystems um betriebliche Aufstiegspositionen.

In unserem Beitrag wollen wir den Stellenwert beruflicher Qualifikationsprofile für die Erstausbildung, für die Lernformen und Kompetenzentwicklung sowie für die internen Karrierepfade und damit die Führungskräfterekrutierung der Industriekaufleute analysieren. Dabei konzentrieren wir uns auf die Investitionsgüterindustrie als wichtigstem Einsatzort der Industriekaufleute und kontrastieren sie mit den Entwicklungen der Privatwirtschaft insgesamt. Unsere Analyse ruht auf zwei Säulen: einer Auswertung der Qualifikationsprofile und Aufstiegs- und Karrieremuster von Industrieangestellten - unter denen die Industriekaufleute die größte Gruppe stellen - auf Basis des Sozio-oekonomischen Panels (SOEP) sowie zwei Fallstudien bei den beiden großen

ABB. 1

\section{Entwicklung der Neuabschlüsse von Ausbildungsverträgen für den Beruf Industriekaufmann/-frau 1995 - 2011}

Angaben in Tsd.

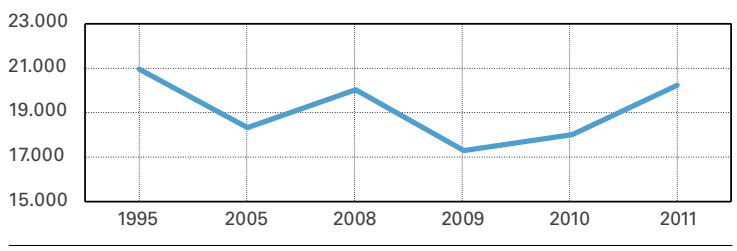

Quelle: BIBB (2012a); Berechnung und Darstellung der Autoren.
Automobilendherstellern VW und Ford. ${ }^{1}$ Die Konzentration auf diese beiden Großkonzerne bedeutet einerseits, dass unsere Ergebnisse nicht direkt auf die vielen kleineren Unternehmen übertragbar sind. Andererseits sind die Großunternehmen der Automobilindustrie häufig Vorreiter für Entwicklungen, die später von anderen Unternehmen übernommen werden. Vor diesem Hintergrund werfen die Fallstudien Licht auf den Organisationswandel und die Personalstrategien, die in den Unternehmen den Stellenwert beruflicher Qualifikationsprofile bestimmen und verändern.

\section{Berufliche Fachlichkeit und Erstaus- bildung}

Welchen Stellenwert haben die Ausbildungen zum Industriekaufmann bzw. zur Industriekauffrau für Schulabgänger im Zeitverlauf? Abbildung 1 zeigt, dass die Ausbildung zum Industriekaufmann bzw. zur Kauffrau im Zeitverlauf ihre Attraktivität für Schulabgänger beibehalten hat. Für diesen Ausbildungsgang ist festzustellen, dass sich die Anzahl der Neuabschlüsse von Ausbildungsverträgen in den 13 Jahren zwischen 1995 und 2008 nur geringfügig reduziert hat. Im Krisenjahr 2009 ist die Anzahl der Neuabschlüsse jedoch deutlich gesunken und liegt erst im Jahr 2011 wieder auf dem Niveau von 2008.

Wie Abbildung 2 zeigt, ist bei den Industriekaufleuten ein deutlicher Trend zur Höherqualifikation zu konstatieren. So haben im Jahr 2011 fast drei Viertel aller Industriekaufleute Abitur. Unklar bleibt, worauf sich diese Veränderungen zurückführen lassen. Legen die Firmen tatsächlich mehr Wert darauf, formal höher Qualifizierte, d. h. Abiturienten, einzustellen? Oder handelt es sich dabei um einen reinen Skaleneffekt, d. h. der steigende Anteil an Abiturienten bei den Kaufleuten ist eine Folge ihres steigenden Anteils in den Schulabgangskohorten? Wie auch immer diese Fragen beantwortet werden, so lässt sich in jedem Fall sagen, dass die traditionelle Berufsausbildung auch für Abiturientinnen und Abiturienten attraktiv bleibt. Die Industriekaufleute liefern damit einen Beleg für die These, dass der Trend zur Höherqualifikation bei der schulischen Ausbildung in der Konsequenz nicht dazu führt, dass die duale Ausbildung ihren Status als Anker des deutschen Beschäftigungssystems verliert (Bosch 2009).

Anhand des SOEP lassen sich die Qualifikationsprofile von einfachen und mittleren Angestellten in der Industrie

1 Grundlage der Fallstudien sind insgesamt zwölf leitfadengestützte Experteninterviews mit Verantwortlichen für Personal(entwicklung), Betriebsräten und kaufmännischen Angestellten sowie ergänzende Auswertungen von Dokumenten der Unternehmen, etwa Ausbildungsbroschüren, Fortbildungskatalogen und Homepages. 
nachzeichnen, unter denen die Industriekaufleute die wichtigste Beschäftigtengruppe stellen. Für die Darstellung der Qualifikationsprofile verwenden wir die vom SOEP bereitgestellte Variable „Stellung im Beruf“, die die folgenden drei Unterteilungen anbietet: Angestellte mit einfacher Tätigkeit (= einfache Angestellte), Angestellte mit qualifizierter Tätigkeit (= mittlere Angestellte) und Angestellte mit hochqualifizierter Tätigkeit oder Leitungsfunktion und Angestellte mit umfassenden Führungsaufgaben (= Führungskräfte). Dabei nutzen wir das Konzept des Bildungstypus. Dieses Konzept differenziert zwischen dem „rein beruflichen“, dem sogenannten „beruflich-akademischen“ Mischtypus und dem „rein akademischen Bildungstypus“, die sich voneinander durch eine unterschiedliche Zusammensetzung von beruflichen und den im allgemeinen Bildungssystem erzielten Abschlüssen unterscheiden (Franz/Voss-Dahm 2011, S. 3). Ein Überblick über die genaue Operationalisierung des Bildungstypus findet sich in Übersicht 1 .

Abbildung 3 stellt die Entwicklung der Bildungsprofile für einfache und mittlere Angestellte der Privatwirtschaft, des Verarbeitenden Gewerbes und für die Investitionsgüterindustrie dar. Auffällig ist, dass der Anteil derjenigen Beschäftigten, die einen rein beruflichen Abschluss aufweisen, zwar rückläufig ist, aber auch im Jahr 2010 in der Privatwirtschaft insgesamt noch bei knapp 87 \% lag. In den rund 35 Jahren des Beobachtungszeitraums beträgt die Veränderung also knapp neun Prozentpunkte. Auffällig ist auch, dass die Verschiebung der Anteile des rein beruflichen Bildungstypus im Bereich der Investitionsgüterindustrie gebremst, eventuell sogar bereits abgeschlossen zu sein scheint. Bereits 1984 lag der Anteil Angestellter mit einer rein beruflichen Ausbildung um vier Prozentpunkte unter dem der Privatwirtschaft. Insgesamt zeigt sich, dass der Stellenwert der beruflichen Ausbildung in Deutschland nach wie vor sehr hoch ist, zumindest was die Qualifikation der einfachen und mittleren Angestellten betrifft. Dabei scheint die Investitionsgüterindustrie einen Sonderfall darzustellen: Anders als in der Privatwirtschaft insgesamt hat der Stellenwert der beruflichen Ausbildung in der Investitionsgüterindustrie wieder an Bedeutung gewonnen.

Auch im Rahmen unserer Fallstudien sind wir der Frage nach dem Stellenwert der beruflichen Ausbildung nachgegangen. Konkret hat uns interessiert, welche Einstiegsmöglichkeiten für kaufmännische Angestellte die Unternehmen anbieten, welche Schulabschlüsse Voraussetzung für eine Ausbildung im kaufmännischen Bereich sind und ob bzw. wie sich die Anforderungen an kaufmännische Angestellte verändern.

Der Automobilhersteller Volkswagen (VW) hat sich im Rahmen seiner „Strategie 2018“ ehrgeizige Ziele gesetzt: Innerhalb von zehn Jahren will das Unternehmen technische Innovationen vorantreiben, um bei Kundenzufriedenheit und Qualität weltweit führend zu werden. Der Konzern will den Absatz und die Umsatzrendite beträchtlich steigern und sich zu einem „Top-Arbeitgeber“ entwickeln. Unsere Gesprächspartner bestätigen, dass diese strategische Ausrich-
ABB. 2

\section{Schulische Vorbildung von Industriekaufleuten im Zeitvergleich 1993 - 2011}
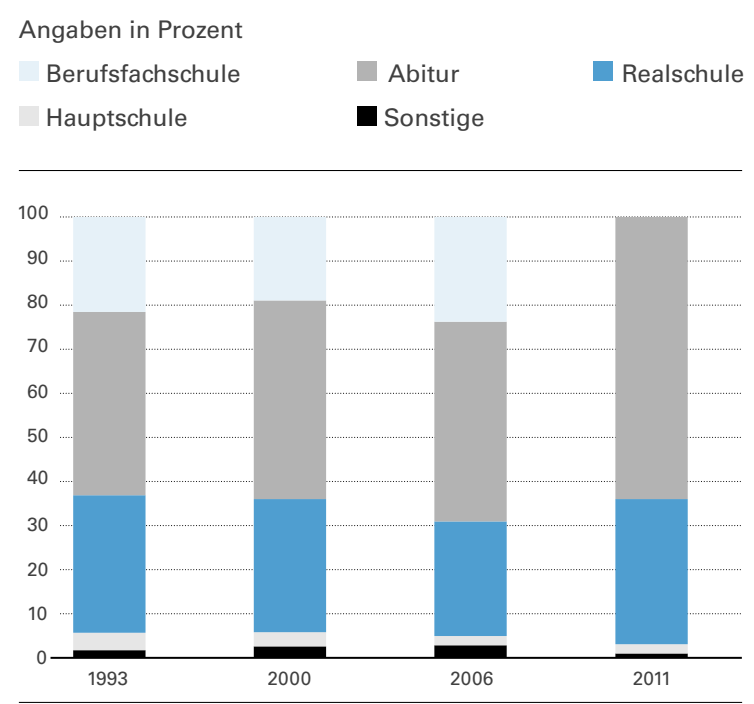

Quelle: BIBB (2012b); Berechnung und Darstellung der Autoren. tung zu einer steigenden Bedeutung des Themas Kompetenz geführt hat und im Unternehmen ein umfassendes Kompetenz- und Personalentwicklungssystem aufgebaut wird. Grundlage dafür ist die Bildung von Berufsfamilien, zu denen alle Mitarbeiterinnen und Mitarbeiter zählen, „die Tätigkeiten auf der Basis verwandter Fachkompetenzen

\section{ÜBERSICHT 1}

\section{Zusammensetzung der Bildungstypen}

\begin{tabular}{|c|c|c|c|}
\hline $\begin{array}{l}\text { Höchster } \\
\text { Bildungsabschluss }\end{array}$ & $\begin{array}{l}\text { Beruflicher } \\
\text { Abschluss durch }\end{array}$ & & $\begin{array}{l}\text { Akademischer } \\
\text { Abschluss durch }\end{array}$ \\
\hline \multirow{6}{*}{$\begin{array}{l}\text { Beruflicher } \\
\text { Bildungstyp }\end{array}$} & Lehre & & \\
\hline & Berufsfachschule & & \\
\hline & $\begin{array}{l}\text { Schule Gesundheits- } \\
\text { wesen (bis 1999) }\end{array}$ & & \\
\hline & Fachschule, Meister & & \\
\hline & Beamtenausbildung & & \\
\hline & Sonstiger Abschluss & & \\
\hline \multirow{6}{*}{$\begin{array}{l}\text { Beruflich- } \\
\text { akademischer } \\
\text { Bildungstyp }\end{array}$} & Lehre & \multirow{6}{*}{ und } & \multirow{6}{*}{$\begin{array}{l}\text { Abschluss einer Fachhochschule/ } \\
\text { Universität oder Promotion }\end{array}$} \\
\hline & Berufsfachschule & & \\
\hline & $\begin{array}{l}\text { Schule Gesundheits- } \\
\text { wesen (bis 1999) }\end{array}$ & & \\
\hline & Fachschule, Meister & & \\
\hline & Beamtenausbildung & & \\
\hline & Sonstiger Abschluss & & \\
\hline $\begin{array}{l}\text { Rein akademischer } \\
\text { Bildungstyp }\end{array}$ & & & $\begin{array}{l}\text { Abschluss einer Fachhochschule/ } \\
\text { Universität oder Promotion }\end{array}$ \\
\hline
\end{tabular}




\section{Entwicklung der Bildungsprofile für einfache und mittlere Angestellte in} Privatwirtschaft, verarbeitendem Gewerbe und Investitionsgüterindustrie Angaben in Prozent

beruflicher Bildungstyp — beruflich-akademischer Bildungstyp —akademischer Bildungstyp

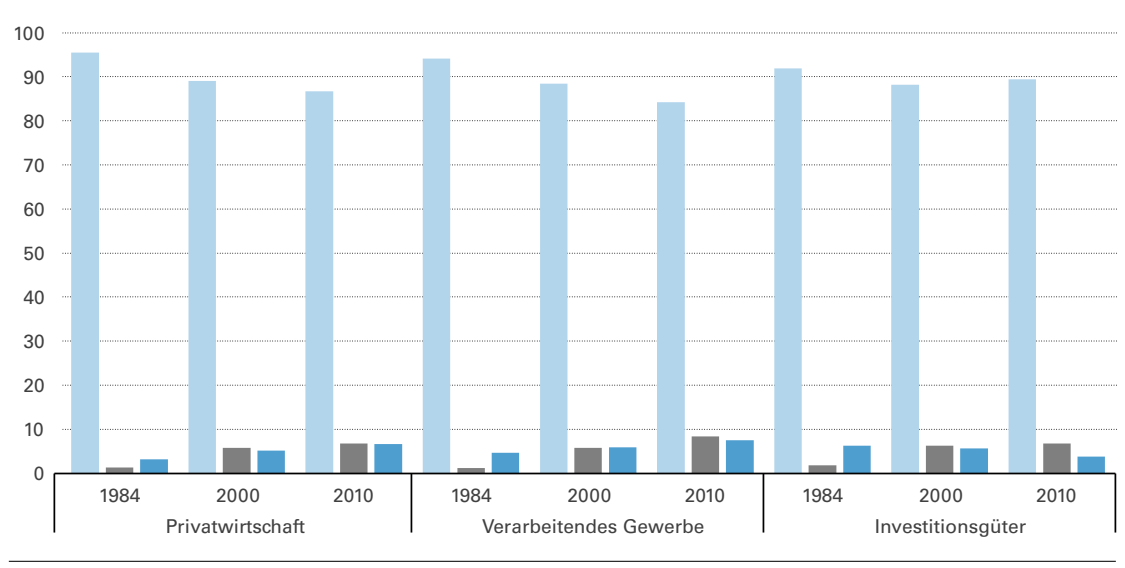

Quelle: SOEP; Berechnung und Darstellung der Autoren.
Laut Angaben der Personalabteilung haben kaufmännische Auszubildende bei VW inzwischen etwa zu zwei Dritteln Abitur und zu einem Drittel einen Realschulabschluss. Allerdings werden auch weiterhin Realschulabgänger eingestellt, um mit ihnen einfachere Angestelltenfunktionen besetzen zu können. Zudem bietet VW die Möglichkeit, in den Beruf über ein duales Studium einzusteigen, also eine praxisorientierte berufliche Ausbildung im dualen Ausbildungssystem mit einer akademischen im Hochschulsystem zu kombinieren. Im kaufmännischen Bereich offeriert das Unternehmen fünf duale Studiengänge. Im Jahr 2012 haben 231 Auszubildende ein duales Studium im Unternehmen abgeschlossen, im Vorjahr waren es 184 (www.volkswagenkarriere.de). Bei aller Vorsicht kann man also sagen, dass das duale Studium an Bedeutung zu gewinnen scheint. Auch die Teilnehmenden an dualen Studiengängen lernen bereits während der Ausbildungsphase die Abläufe im Unternehmen kennen und bauen interne Netzwerke auf.

Unsere Gesprächspartner beschreiben, dass sich die Anforderungen an (Industrie)kaufleute bei VW - unabhängig von der Art ihrer Ausbildung - verändern: Sozialkompetenzen gewinnen an Bedeutung, da die Beschäftigten auf so gut wie allen Positionen eng mit anderen zusammenarbeiten müssen. Aufgrund der internationalen Ausrichtung des Konzerns spielen auch sprachliche und interkulturelle Kompetenzen eine zentrale Rolle.

Beim Automobilhersteller Ford hat der Stellenwert der Berufsausbildung im Konzern in den letzten Jahren deutlich zugenommen. Während in dem amerikanischen Unternehmen die Berufsausbildung vorher zwar akzeptiert, aber eher als exzentrische institutionelle Besonderheit der deutschen Standorte wahrgenommen wurde, gilt die Berufsausbildung inzwischen als Leitbild auch für andere Standorte des Konzerns. Die breite Spanne, die an Standorten ohne berufliche Ausbildung zwischen Anlerntätigkeiten auf der einen sowie höheren schulischen und akademischen Abschlüssen auf der anderen Seite liegt, wird zunehmend als Problem empfunden. Tätigkeiten mittleren Qualifikationsniveaus lassen sich so nur schwer besetzen oder können gar nicht erst in der Organisation verankert werden. Und die Trennung zwischen Ausführenden und Experten bleibt zwangsläufig groß. Deshalb wird an den nicht-deutschen Standorten auch zunehmend damit experimentiert, Elemente der beruflichen Fachlichkeit einzuführen und zu vermitteln.

Der hohe Stellenwert der beruflichen Erstausbildung in Deutschland wird dadurch unterstrichen, dass Personalbereich und Betriebsrat gemeinsam Anfang der 1990er Jahre einen Aus- und Weiterbildungsverein (FAW) gegründet haben, mit dem Ziel, die Ausbildung zu verbessern und effizienter zu gestalten. Die zentrale Aufgabe des FAW besteht darin, eine qualifizierende Berufsausbildung sowohl für die Auszubildenden in den Ford-Werken als auch für verschiedene Joint Venture- und Zulieferunternehmen zu organisieren. Das Bildungsangebot umfasst neben der Übernahme der Berufsausbildung ein umfangreiches Angebot im Bereich Training, Beratung und Coaching. Die schuli- 
schen Vorqualifikationen für die Ausbildung der Industriekaufleute haben sich in den letzten Jahrzehnten auch bei Ford deutlich verändert: Wurden früher vorwiegend Realschüler oder Hauptschüler mit Handelsschulabschluss als Auszubildende eingestellt, so dominieren inzwischen auch hier die Abiturienten. Für sie ist der Beruf Industriekaufmann/-kauffrau neben dem Beruf des Bank- oder Versicherungskaufmanns die Alternative zum Studium. In der Ausbildung werden seitens des Unternehmens neben dem fachlichen Wissen weitere praxisorientierte Kenntnisse und Fähigkeiten vermittelt: „Zum Beispiel viel mehr an ITKenntnissen, als das Berufsbildungsgesetz uns das vorschreibt" (Personalverantwortlicher). Begründet werden diese zusätzlichen Module mit der Notwendigkeit, die Ausbildungsinhalte an neue Anforderungen anzupassen. Diese Anforderungen werden in der kaufmännischen Ausbildung besonders deutlich, weil hier die Auszubildenden von Beginn an in den Fachbereichen eingesetzt werden.

Neben der Berufsausbildung bietet auch Ford seit etwa zehn Jahren ein duales Studium als Kombination aus der dreijährigen kaufmännischen Ausbildung zum Industriekaufmann und einem Wirtschaftsstudium an. Jedes Jahr werden nach Angaben des FAW zwölf Auszubildende dafür eingestellt. Diese Zahl ist langjährig stabil, und die Chancen, nach der Ausbildung im Unternehmen zu verbleiben, sind sehr hoch. Mindestanforderung an die Bewerberinnen und Bewerber für das duale Studium ist ein gutes Abitur. $\mathrm{Zu}$ gleich aber werden, wie auch bei VW, bewusst weiterhin Realschüler eingestellt, um mit ihnen auch die einfacheren Tätigkeiten im kaufmännischen Bereich besetzen zu können. Extern eingestellte Hochschulabsolventen durchlaufen ein sogenanntes Graduates-Programm. Der Einstieg auf diesem Wege ist jedoch nur begrenzt möglich und er ist in den letzten Jahren aufgrund der wirtschaftlich schwierigen Situation weiter eingeschränkt worden.

Sowohl bei VW als auch bei Ford kann also von einem Bedeutungsverlust der dualen Ausbildung keine Rede sein. Bei VW sind der Aufbau einer „Spitzenmannschaft" und damit die Kompetenzentwicklung zu einem Kernelement der Unternehmensstrategie geworden. Bei Ford erfährt die deutsche Berufsausbildung mittlerweile internationale Anerkennung im Konzern. Darüber hinaus ist in beiden Unternehmen zu beobachten, dass die berufliche Ausbildung zunehmend im Rahmen eines dualen Studiums mit (Fach) Hochschulabschlüssen kombiniert wird.

\section{Karriere- und Kompetenzentwicklung}

Wie steht es um die Entwicklungsperspektiven beruflich Qualifizierter? Die Industrie- und Handelskammer bietet mit dem Fachwirt Industrie für Absolventen der dualen Ausbildung eine Aufstiegsfortbildung an, die auf den in der Erstausbildung erworbenen Kenntnissen aufbaut und sys- tematisch auf das mit einem Aufstieg verbundene erweiterte Aufgabenfeld vorbereitet. Die Teilnahmezahlen dieser Fortbildungen haben sich zwischen den Jahren 1992 und 1996 deutlich verringert (- 57 \% von 3.544 auf 2.008). Danach haben sie sich auf diesem Niveau stabilisiert und sind seit 2004 auf 2.137 Teilnehmer im Jahr 2006 angestiegen (BIBB 2008). Im Jahr 2009 verzeichnet der Deutsche Industrie- und Handelskammertag (DIHK) 2.281 Teilnahmen an Fortbildungsprüfungen. Eine neue Erfassungsmethode in den Folgejahren macht einen direkten Vergleich der Zahlen nicht mehr möglich. Nach der neuen Erhebungsmethode haben im Jahr 20101.747 und im Jahr 20111.795 Teilnehmer an den Fortbildungsprüfungen zum Industriefachwirt teilgenommen, bestanden haben allerdings nur 1.262 (DIHK 2012). Demnach lässt sich zumindest bis in das Jahr 2009 feststellen, dass berufliche Qualifikationen und Kompetenzen nach einer Phase der Abwertung in den 1980er und 90er Jahren wieder an Boden und Bedeutung gewinnen. Darin ähnelt diese Entwicklung derjenigen der Erstausbildung. Inwieweit der ab 2010 festzustellende Rückgang der Teilnehmerzahlen allein der Umstellung in der Erfassungsmethode geschuldet ist oder Anzeichen sinkender Attraktivität des Fachwirts darstellt, lässt sich aus heutiger Sicht allerdings kaum beurteilen.

Welcher Stellenwert wird Qualifizierung und Kompetenzentwicklung in den untersuchten Unternehmen eingeräumt? Welche Qualifizierungen werden angeboten oder unterstützt, welche Lernformen finden dabei Anwendung?

Bei VW wurde in den letzten Jahren ein unternehmensweites systematisches Kompetenz- und Personalentwicklungssystem aufgebaut. Zentrales Element und Grundlage der Personalentwicklung sind Mitarbeitergespräche und Kompetenzprofile. Im Rahmen von jährlichen Mitarbeiterund Qualifizierungsgesprächen mit dem oder der direkten Vorgesetzten sprechen alle Beschäftigten außer über die leistungsorientierte Vergütung auch über persönliche Entwicklungsmöglichkeiten. Zur Orientierung dienen dabei sogenannte Kompetenzprofile, die für gleichartige Positionen entwickelt wurden. Diese Kompetenzprofile decken in erster Linie fachliche Fähigkeiten ab, aber auch überfachliche Kompetenzen wie zum Beispiel das Kommunikationsund Kooperationsverhalten. Qualifizierungsangebote werden parallel zur Systematik der Kompetenzprofile offeriert. Beschäftigte und Vorgesetzte können also gemeinsam das Kompetenzprofil studieren, diskutieren, wo Defizite bestehen, und das individuell passende Weiterbildungsangebot auswählen. Umgekehrt dienen die Gespräche aber auch dazu, neue Qualifikationsbedarfe zu ermitteln, die dann von den Weiterbildungsanbietern im Unternehmen realisiert werden.

Im Rahmen des Mitarbeitergesprächs gibt der oder die Vorgesetzte auch eine sogenannte Potenzialeinschätzung ab. Diese eröffnet drei Optionen: Die Weiterentwicklung auf der aktuellen Position, also den zumindest temporären Aufschub eines weiteren Karriereschrittes; einen horizontalen Entwicklungsschritt in einen anderen Bereich; oder 
die Empfehlung eines vertikalen Schrittes, der mit einem hierarchischem Aufstieg verbunden ist. Qualifizierung kann (bzw. muss in einigen Fällen) ein Bestandteil aller drei Optionen sein. Das Unternehmen bietet seinen Beschäftigten vielfältige Möglichkeiten zur Weiterqualifizierung: Neben der Möglichkeit zur Teilnahme an unternehmensinternen Weiterbildungsangeboten, die von Vorträgen über „Vormachreihen“, klassische Seminare und modulare Programme bis hin zu Patensystemen reichen, gibt es verschiedene Programme, auf die sich die Beschäftigten bewerben können. So können Berufsanfänger seit 2006 im Programm „Wanderjahre“ für in der Regel zwölf Monate bei einer Konzerngesellschaft Auslandserfahrungen sammeln. Eine besondere Zielsetzung der „Wanderjahre“ ist, dass die Teilnehmer den Auslandsaufenthalt nutzen, um sich ein internationales Netzwerk innerhalb des Konzerns aufzubauen und gleichzeitig ihr Fachwissen zu erweitern. Die Teilnahme an anerkannten beruflichen Weiterbildungen wird in Form von Fachstipendien gefördert. In Zusammenarbeit mit den Industrie- und Handelskammern bietet das Unternehmen verschiedene anerkannte Fortbildungsmaßnahmen an, zum Beispiel „Ausbildung der Ausbilder“oder „Office-Manager“. Mitarbeiterinnen und Mitarbeiter, die sich in Form eines Studiums weiterqualifizieren möchten, unterstützt das Unternehmen mit einer Studienförderung. Welche Studiengänge gefördert werden, wird jedes Jahr neu entschieden. Dabei haben Bewerber, die einen internationalen Studiengang anstreben, besonders gute Chancen. Für jede Berufsfamilie wurde eine eigene Akademie gegründet, die alle Qualifizierungsmaßnahmen für die jeweilige Berufsfamilie bündelt. Die Mitglieder einer Berufsfamilie lernen miteinander und voneinander, unabhängig von ihrer Ausbildung (z. B. im Personalbereich: Juristen und Psychologen) und ihrem Status. Nur wenn keine internen Referenten zur Verfügung stehen, werden externe gesucht. Die Qualifizierung bei Volkswagen wird also zunehmend auf die konkreten Arbeits- bzw. Geschäftsprozesse ausgerichtet und damit unternehmensspezifischer bzw. individueller. Moderne Qualifizierungsformen wie die erwähnten „Vormachreihen" setzen diesen Ansatz methodisch um.

Bei Ford wird die Weiterbildung über den FAW organisiert. Das Unternehmen bietet seinen Mitarbeitern eine Vielzahl an internen Weiterbildungsmaßnahmen an, auch wenn die Mittel im Bereich der Weiterbildung deutlich reduziert worden sind. Grund hierfür ist die schwache wirtschaftliche Entwicklung des Unternehmens in den letzten Jahren. Weiterbildung erfolgt meist kostengünstig über interne Trainings zur Kompetenzentwicklung. Auf diese Weise sollen zwei Fliegen mit einer Klappe geschlagen werden. Auf der einen Seite sollen Kosten gesenkt werden, auf der anderen Seite soll aber auch interne Expertise genutzt und ausgeschöpft werden, um einen problemnahen Kompetenztransfer zu ermöglichen. Dazu gehören Konzepte wie Coaching, Teaching und Shadowing: Führungskräfte und Experten begleiten Beschäftigte, halten interne Vorträge oder lassen sich bei ihrer Arbeit auf die Finger schauen. Diese internen
Weiterbildungsmaßnahmen können jedoch nur funktionieren, wenn die Abteilungen zusammenarbeiten und die Kompetenzträger auch für die interne Weiterbildung freistellen.

Der Blickwinkel der Weiterbildung hat sich nach Aussage der Expertinnen und Experten grundlegend verschoben. Stand früher das Trainingsprogramm an erster Stelle, so ist es nun der Kompetenzbedarf: Nicht mehr das Trainingsangebot prägt die individuelle Kompetenzentwicklungsplanung, sondern die möglicherweise fehlenden, ausbaufähigen oder zu aktualisierenden Kompetenzen. Eine wichtige Rolle im Kompetenzerwerb spielen die Trainingsund Entwicklungspläne, die für jeden Beschäftigten definiert werden. Zu Beginn einer Tätigkeit gibt es zunächst eine Einarbeitungsphase in dem neuen Beschäftigungsbereich sowie bereichsübergreifende Einführungsveranstaltungen und Trainings. Im Anschluss daran wird mit dem oder der jeweiligen Vorgesetzten ein individueller Kompetenzentwicklungsplan entworfen und in Zusammenarbeit mit einem Personalentwicklungskomitee umgesetzt. Die Betreuung durch das Entwicklungskomitee soll der professionellen Entwicklung der einzelnen Mitarbeiter dienen und langfristige Karrierechancen gewährleisten. Über die Kompetenzentwicklungspläne soll die Förderung individuell auf den Beschäftigten abgestimmt werden.

Der Wandel der Lernformen wird von den Experten in direkten Zusammenhang zur Veränderung der Kompetenzanforderungen gebracht. Dabei benennen die Experten zumindest drei zentrale Entwicklungen: eine wachsende Komplexität der Arbeitsaufgaben durch den Wegfall von Routinearbeiten und die Ausdünnung der Personaldecke; neue Kompetenzanforderungen durch IT-gestützte Prozesse und die Internationalisierung in Beschaffung und Vertrieb; und schließlich die Zunahme der Arbeitsverdichtung durch Personalabbau, die Integration von Tätigkeiten und technisch gestützte dauernde Erreichbarkeit. Fachliche Qualifikationen behalten ihren hohen Stellenwert, hinzu treten neue Kompetenzanforderungen mit Blick auf Problemlösungskompetenzen, Team- oder Kommunikationsfähigkeit. Zusätzlich wachsen die Kompetenzanforderungen mit Blick auf die Matrixorganisation des Unternehmens. Hier überlappen sich regionale, zentrale oder produktbezogene Zuständigkeiten, was gerade im kaufmännischen Bereich zur Folge hat, dass ein Beschäftigter für mehrere Organisationsbereiche arbeitet. Als Mitarbeiter der Beschaffung ist er beispielsweise an das zentrale Einkaufsressort der Konzernmutter angeschlossen, dabei aber zuständig für einen bestimmten Produktbereich und gleichzeitig einem deutschen Standort zugeordnet. Dies hat auch zur Folge, dass fachliche und disziplinarische Vorgesetztenfunktionen auseinanderfallen.

In beiden Unternehmen lässt sich ein Wandel von Kompetenzentwicklung und Lernformen feststellen. Die Kompetenzentwicklung erhält durch regelmäßige Personalentwicklungsgespräche einen individuelleren Zuschnitt. In methodischer Hinsicht werden die unterschiedlichsten Lernformen eingesetzt, sowohl bei VW als auch bei Ford gewinnen jedoch unternehmensinterne, informelle und arbeits- 
bzw. geschäftsprozessnahe Formen der Qualifizierung an Bedeutung. Bei der Entscheidung für diese Lernformen spielen in beiden Unternehmen Kostenaspekte eine Rolle, die uns jedoch bei Ford expliziter genannt wurden als bei VW.

\section{Karrierepfade}

Für die Analyse der Karrierepfade zeichnen wir Qualifikationsprofile, wie wir sie oben für die einfachen und mittleren Angestellten analysiert haben, für die Gruppe der Führungskräfte nach. Das SOEP unterscheidet bei Führungskräften zwei Gruppen: „Angestellte mit hochqualifizierter Tätigkeit oder Leitungsfunktion“ und „umfassende Führungsaufgaben“, die wir jedoch analog zu anderen Analysen (Franz 2011; Holst/Busch 2010) in dem Konstrukt „Führungskraft“ zusammenführen.

Wie Abbildung 4 zeigt, besaß in der Privatwirtschaft im Jahr 1984 die knappe Mehrheit der Führungskräfte noch einen rein beruflichen Abschluss - nur jede dritte Führungsposition wurde von einer Person mit einem rein akademischen Bildungshintergrund besetzt. Einen gemischten Ausbildungshintergrund wies etwas mehr als jede zehnte Führungskraft auf. Im Jahr 2010 dominierte hingegen der akademische Bildungstyp mit einem Anteil von rund $44 \%$, während die Verbreitung des beruflichen Bildungstypus auf nur noch gut $30 \%$ abgesunken ist. Zudem hat sich der Anteil des Mischtyps beruflicher und akademischer Ausbildung auf über $23 \%$ mehr als verdoppelt. Diese Entwicklungen zuungunsten des beruflichen Bildungstyps sind in der Investitionsgüterbranche noch stärker konturiert. Zunächst ist festzustellen, dass bereits im Ausgangsjahr 1984 der Anteil derjenigen Führungskräfte, die einen rein beruflichen Ausbildungshintergrund besaßen, mit $46 \%$ deutlich geringer war als in der Privatwirtschaft insgesamt. Zudem zeigt sich im Zeitvergleich, dass der Anteil der Führungskräfte mit rein beruflichen Abschlüssen in der Investitionsgüterindustrie auch stärker gesunken ist als in der Privatwirtschaft insgesamt. Parallel dazu ist der Anteil der Führungskräfte, die zu dem rein akademischen Bildungstypus zählen, in der Investitionsgüterindustrie $\mathrm{zu}$ jedem Untersuchungszeitpunkt der höchste. Zugleich wird der beruflich-akademische Mischtyp im Zeitvergleich immer wichtiger. Während in der Privatwirtschaft insgesamt im Jahr 2010 knapp jede vierte Leitungsposition mit einer Person, die sowohl einen beruflichen als auch einen akademischen Ausbildungshintergrund aufweisen kann, besetzt ist (+12 Prozentpunkte im Vergleich zu 1984), ist es in der Investitionsgüterindustrie sogar fast jede dritte (+13,5 Prozentpunkte). Damit spielen berufliche Qualifikationen sogar eine wachsende Rolle in Kombination mit akademischen Abschlüssen. In der Summe haben mehr als $50 \%$ der Führungskräfte in der Investitionsgüterindustrie eine berufliche Ausbildung, aber unter den Vorzeichen einer akademischen Erweiterung.
ABB. 4

\section{Entwicklung der Bildungsprofile für Führungskräfte in Privatwirtschaft, verarbeitendem Gewerbe und Investitionsgüterindustrie}

Angaben in Prozent

$\square$ beruflicher Bildungstyp $\square$ beruflich-akademischer Bildungstyp $\square$ akademischer Bildungstyp

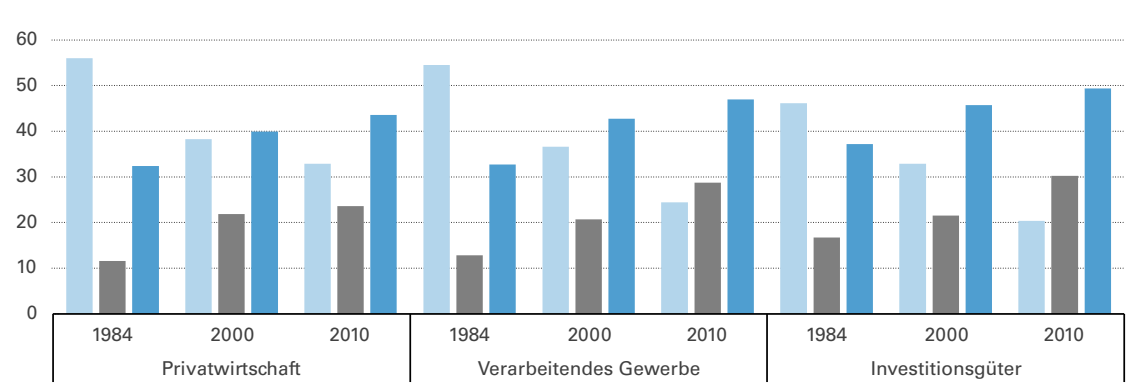

Quelle: SOEP; Berechnung und Darstellung der Autoren.

Die Karrierepfade von Industriekaufleuten haben wir auch in unseren Fallstudien thematisiert. Dabei sind wir den Fragen nachgegangen, welche Instrumente Entwicklungsentscheidungen und -wege regeln und unterstützen und welche Entwicklungsmöglichkeiten Kaufleute in der Industrie mit beruflicher Ausbildung in den Unternehmen haben.

Bei VW wurde seit 2007 ein systematisches Auswahlverfahren für die Führungslaufbahn im Unternehmen entwickelt: Im Anschluss an die Potenzialeinschätzung des Vorgesetzten im Mitarbeitergespräch nehmen die Kandidaten an einem mehrstufigen Verfahren teil. Dabei gilt das „Mehraugenprinzip“, das den Einfluss der direkten Vorgesetzten auf die weitere Entwicklung ihrer Mitarbeiter begrenzen soll. Dieses stark systematisierte und strukturierte Verfahren der Führungskräfteauswahl soll Transparenz für alle Beteiligten herstellen. Die Hierarchien bei Volkswagen sind allerdings vergleichsweise flach, sodass nur eine begrenzte Anzahl von Aufstiegspositionen zur Verfügung steht. Im Gegenzug wird verstärkt auf Fachkarrieren gesetzt, also auf Entwicklungen entlang fachlicher Herausforderungen ohne Führungsverantwortung, aber mit Aussichten auf ein höheres Einkommen, neue Titel und mehr Gestaltungsspielraum. Anders als für die vertikalen Laufbahnen existieren für Fachlaufbahnen im Unternehmen jedoch keine zentralen Auswahlverfahren. Die Frage nach den Entwicklungs- und Karrieremöglichkeiten von Angestellten mit beruflicher Ausbildung beantworten unsere Interviewpartner dennoch grundsätzlich positiv. Ein Gesprächspartner erläutert: „Immer wenn man sich für etwas spezialisiert, ... kann man sich auch relativ gut entwickeln. Und die Möglichkeit gibt es auch für jemanden, der eine mittlere Reife hat und eine Ausbildung zum Industriekaufmann.“ Allerdings ergänzt er einschränkend, dass „die Fälle seltener [werden], weil es doch ganz einfach so ist, dass wir uns einem Wandel unterzogen haben." 
Bei Ford sind die Entwicklungen ähnlich. Die Personalentwicklungskomitees, die in den Betreuungsbereichen eingerichtet wurden, haben die Aufgabe, Fragen der Weiterentwicklung und des Karrierefortschritts für den individuellen Beschäftigten zu entscheiden. Die Komitees sollen das Mehraugenprinzip sicherstellen und die Abhängigkeit der Karriereentwicklung von der Entscheidung eines Vorgesetzten reduzieren. Der Vorteil dieses Verfahrens besteht nach Aussage der Experten vor allem darin, über die Intersubjektivität der Bewertung auch die Versachlichung und Objektivierung einer Personalentscheidung fördern zu können.

Fachliche Spezialisierung als Voraussetzung für die Karriereentwicklung hat bei Ford an Bedeutung verloren. In diesem Punkt hat nach Aussage der Experten in den letzten Jahren eine Annäherung an amerikanische Managementpraktiken stattgefunden. Entscheidend sind zwei Faktoren: dass eine berufliche Vorbildung besteht und dass sie ergänzt wurde um einen Bachelorabschluss. Damit sind die formalen Voraussetzungen für den Aufstieg im Unternehmen erfüllt. Anders als früher gibt es konkrete Karrierefahrpläne für die Beschäftigten innerhalb des Unternehmens aber nicht mehr. Die Entscheidungen werden nun stärker an die Leistung als an formale Qualifikationen gebunden, und die Bewertung wird in intersubjektive Verfahren eingebettet. Zugleich hat sich auch bei Ford die Zahl der Führungspositionen im Gefolge des Abbaus der Hierarchieebenen verringert. Aus Sicht der Experten hat dies allerdings nicht dazu geführt, dass die Karrieremöglichkeiten entscheidend beschnitten worden sind. Dafür ist zum einen die wachsende Bedeutung horizontaler Karriereschritte verantwortlich. Und zum anderen spielen Netzwerke eine immer größere Rolle. Der Kreis der Bezugspersonen hat sich durch funktionsübergreifende Prozesse und die wachsende Zahl internationaler Kontakte deutlich vergrößert: „Ich würde mal so sagen, die Perspektiven sind größer, weil es viel mehr Möglichkeiten gibt. Es gibt viel mehr Cross Functional Moves“ (Personalverantwortlicher).

Zwar werden also durch den Hierarchieabbau die vertikalen Aufstiegsperspektiven deutlich begrenzt, doch erwachsen nach Aussage der Experten neue Chancen aus dem demografischen Wandel. Diese Entwicklung wird demnach dazu führen, dass Nachwuchs für fachliche und hierarchische Karrieren trotz des Rückgangs der Karrierepositionen knapp wird: „Alleine dadurch sind wiederum die Entwicklungsmöglichkeiten, ich möchte fast sagen um ein Vielfaches gestiegen" (Personalverantwortlicher).

Sowohl bei VW als auch bei Ford wird also auf die Anwendung des Mehraugenprinzips bei Karriereentscheidungen Wert gelegt. In beiden Unternehmen gibt es aufgrund von flacheren Hierarchien weniger Perspektiven für vertikale Karrieren; der betriebliche Aufstieg wird für Industriekaufleute schwerer und steiniger und ist zudem mehr als zuvor mit Leistungsbewertungen verbunden. Demgegenüber haben die Möglichkeiten für horizontale Karrieren zugenommen. Basis für eine Karriere ist in beiden Unternehmen eine berufliche Ausbildung, die um weitere Qualifizierung ergänzt wird - bei Ford explizit in Form eines Bachelor-Abschlusses, bei VW sind unterschiedliche Formen der ergänzenden Qualifizierung verbreitet. Dabei wird der Fachlichkeit der Ausbildung bei Ford tendenziell weniger Bedeutung beigemessen als bei VW. Ein Gesprächspartner bei Ford weist darauf hin, dass Berufseinsteiger aufgrund der demografischen Entwicklung in Zukunft wieder zunehmende Möglichkeiten auch für vertikale Karrieren haben werden. Insgesamt aber lässt sich sagen, dass zumindest derzeit eine Berufsausbildung nach wie vor wichtige Karrierevoraussetzung ist, dies aber verbunden mit weiterbildenden und vor allem akademischen Abschlüssen auf dem Bachelor-Niveau.

\section{Zusammenfassung und Fazit}

Berufliche Qualifikation und Fachlichkeit haben für Industrieangestellte wenig von ihrer Bedeutung eingebüßt. Sie sind nach wie vor eine zentrale Grundlage ihrer Qualifikationsund Karriereentwicklung. Zugleich aber wurden sie aktualisiert und gewandelten Anforderungen angepasst. Diese Entwicklungen lassen sich in allen drei Dimensionen der Berufsfachlichkeit von Industrieangestellten feststellen, wenn auch in unterschiedlicher Intensität. Die duale Erstausbildung ist das zentrale Einstiegsportal für Industriekaufleute. Die externe Personalrekrutierung von Hochschulabsolventen hat nur ergänzenden Charakter und ist weit davon entfernt, die Erstausbildung zu verdrängen. Die zentralen Veränderungen vollziehen sich vielmehr innerhalb der Erstausbildung. Dazu gehört, dass das Niveau der schulischen Vorbildung der Auszubildenden ansteigt und dass neue Kompetenzen an Bedeutung gewinnen. IT-, Sozial- und Reflexionskompetenzen werden wichtiger, ohne dass jedoch fachliche Qualifikationen ihren zentralen Stellenwert verlieren. Schließlich wird die Erstausbildung zunehmend mit (Fach)Hochschulabschlüssen im Rahmen eines dualen Studiums kombiniert, das von den Unternehmen gefördert wird.

Kompetenz ist auch der Schlüsselbegriff für die interne Qualifikationsentwicklung als zweiter Dimension der Berufsfachlichkeit. Der Kompetenzbedarf wird inzwischen individuell und damit passgenauer im Rahmen von Personalentwicklungsgesprächen mit dem Vorgesetzten festgelegt. Hierbei können die Beschäftigten ihre Entwicklungsinteressen einbringen. Bei der Aktualisierung von Berufsfachlichkeit durch Kompetenzentwicklung setzen die Unternehmen verstärkt auf interne Angebote sowie auf arbeitsplatznahe und informelle Lernformen, die auf der Mobilisierung internen Experten- und Führungswissens beruhen. Dafür geben auch finanzwirtschaftliche Zielsetzungen den Ausschlag, die sich in Kürzungen der Weiterbildungsbudgets niederschlagen.

Deutliche Anzeichen für einen Bedeutungsverlust beruflicher Qualifikationen sind hingegen bei den Karrierewegen als dritter Dimension erkennbar. Zunächst ist festzuhalten, dass die Verbindung der Berufsausbildung mit zusätzlichen akademischen Abschlüssen mehr und mehr zur Bedingung 
vertikaler Karrierepfade wird. In diesem Rahmen können bereits Bachelorabschlüsse als Eintrittskarte auch für steile Karrieren genügen. Dabei kommt der alltäglichen Arbeitsleistung der Beschäftigten eine wachsende Bedeutung zu. Hierbei organisieren die Unternehmen intersubjektive Bewertungsverfahren mit dem Ziel, die Personalentwicklung stärker zu verobjektivieren. Insgesamt aber sinkt die Zahl der Aufstiegspositionen in den Unternehmen und damit die Wahrscheinlichkeit eines beruflichen Aufstiegs für die Industriekaufleute. Dies liegt an der Abflachung betrieblicher Hierarchien. In den Unternehmen wird zwar die kompensatorische Funktion horizontaler Entwicklungsperspektiven betont; für die Beschäftigten dürfte diese Kompensation allerdings keinen vollwertigen Ersatz für verlorene Aufstiegschancen bieten. Als Voraussetzung für den Aufstieg haben berufliche Qualifikationen nach wie vor eine große Bedeutung. Allerdings müssen sie mehr und mehr mit Weiterbildungszertifikaten und -aktivitäten sowie, besser noch, einem akademischen Abschluss kombiniert werden. Statt Konkurrenz ist also eher eine Symbiose der Abschlüsse als Bedingung für Karrieren festzustellen. In diese Richtung geht auch der Ausbau dualer Studiengänge, die es in den beiden Fallunternehmen gibt. Wie sich die Unternehmen allerdings unter dem Druck einer weiteren Akademisierung und eines möglichen Nachwuchsmangels im dualen Ausbildungssystem verhalten werden, steht in den Sternen. Es ist jedenfalls nicht auszuschließen, dass sie im Sog solcher Entwicklungen die Orientierung an der beruflichen Fachlichkeit lockern werden. Möglicherweise gelingt es ihnen aber auch, diese Trends in Richtung Erhalt der Fachlichkeit zu beeinflussen.

\section{LITERATUR}

Baethge, M. (2001): Kommunikation und Wissen: Basisqualifikationen moderner Dienstleistungen, in: Sommer, M./Schröder, L./Schwemmle, M. (Hrsg.): Neu Denken - Neu Handeln: Arbeit und Gewerkschaft im digitalen Kapitalismus, Hamburg, S. 99-120

Baethge, M./Baethge-Kinsky, V. (1998): Jenseits von Beruf und Beruflichkeit? Neue Formen von Arbeitsorganisation und Beschäftigung und ihre Bedeutung für eine zentrale Kategorie gesellschaftlicher Integration, in: Mitteilungen aus der Arbeitsmarkt- und Berufsforschung 31 (3), S. 461-472

Baethge, M./Baethke-Kinsky, V./Holm, R./Tullius, K. (2006): Dynamische Zeiten - langsamer Wandel: Betriebliche Kompetenzentwicklung von Fachkräften in zentralen Tätigkeitsfeldern der deutschen Wirtschaft, Projektbericht, Göttingen Bundesinstitut für Berufsbildung (BIBB) (2008): Datenblätter zur Situation der beruflichen Aus- und Weiterbildung, http://www.bibb.de/de/5550.htm, Stand 20.12.2012

Bundesinstitut für Berufsbildung (BIBB) (2012a): Datenblätter 7803 (Bürokaufmann/kauffrau) und 7851 (Industriekaufmann/kauffrau), Stand: 09.11.2012 Bundesinstitut für Berufsbildung (BIBB) (2012b): Datensystem Auszubildende, http://www.bibb.de/de/1108.htm, Stand: 20.12.2012

Boes, A./Kämpf, T. (2010): Offshoring and New Insecurities: Towards New Types of 'White Collar Consciousness' in Globalised Working Environments, in: Work Organisation, Labour and Globalisation 1 (4), S. 104-119

Boes, A./Pfeiffer, S. (2006): Thesen zur Informatisierung der Arbeit: Neue Qualität der Entwicklung, neue Perspektiven für die Arbeitsforschung, in: Dunkel, W./Sauer, D. (Hrsg.): Von der Allgegenwart der verschwindenden Arbeit: Neue Herausforderungen für die Arbeitsforschung, Berlin, S. 33-43

Bosch, G. (2000): The Dual System of Vocational Training in Germany: Is it still a Model?, in:Tremblay, D.-G./Doray, P. (Hrsg.): Vers de nouveaux modes des formation professionelle?, Quebec, S. 91-114

Bosch, G. (2009): The Revitalization of the Dual System of Vocational Training in Germany, in: Bosch, G./Charest, J. (Hrsg.): Vocational Education and Training, S. 137-161

Deutscher Industrie und Handelskammertag (DIHK) (2012): DIHK - Fortbildungsstatistik 2012. Internetdokument, www.dihk.de/ressourcen/downloads/ fortbildung-2011 (letzter Zugriff 25.7.2013)

Dörre, K. (2012): Krise des Shareholder Value? Kapitalmarktorientierte Steuerung als Wettkampfsystem, in: Kraemer, K./Nessel, S. (Hrsg.): Entfesselte Fi- nanzmärkte: Soziologische Analysen des modernen Kapitalismus, Frankfurt a. M./New York, S. 121-144

Dunkel, W./Rieder, K. (2004): Interaktionsarbeit zwischen Konflikt und Kooperation, in: Dunkel, W./Voß, G. G. (Hrsg.): Dienstleistung als Interaktion: Beiträge aus einem Forschungsprojekt, München/Mering, S. 211-226

Faust, M./Jauch, P./Notz, P. (2000): Befreit und entwurzelt: Führungskräfte auf dem Weg zum internen Unternehmer, München/Mering

Franz, C. (2011): Bildungsprofile von Führungskräften: Vielfalt statt Verdrängung, in: Voss-Dahm, D./Mühge, G./Schmierl, K./Struck, O. (Hrsg.): Qualifizierte Facharbeit im Spannungsfeld von Flexibilität und Stabilität, Wiesbaden, S. $187-210$

Franz, C./Voss-Dahm, D. (2011): Ohne Studium (k)eine Führungsposition? Nach wie vor starke Bedeutung von beruflichen Bildungsabschlüssen bei Führungskräften in der Privatwirtschaft, IAQ-Report 2/2011, Duisburg/Essen Finegold, D./Wagner, K. (1999): The German Skill-Creation System and TeamBased Production: Asset or Liability?, in: Culpepper, P. D./Finegold, D. (Hrsg.): The German Skills Machine: Sustaining Comparative Advantage in a Global Economy, New York/Oxford, S. 115-155

Herrigel, G./Sabel, C. F. (1999): Craft Production in Crisis: Industrial Restructuring in Germany During the 1990s, in: Culpepper, P. D./Finegold, D. (Hrsg.): The German Skills Machine: Sustaining Comparative Advantage in a Global Economy, New York/Oxford, S. 77-114

Holst, E./Busch, A. (2010): Führungskräfte-Monitor 2010, in: DIW (Hrsg.): Politikberatung kompakt (56), Berlin

Holtgrewe, U./Voswinkel, S. (2002): Kundenorientierung zwischen Mythos, Organisationsrationalität und Eigensinn der Beschäftigten, in: Sauer, D. (Hrsg.): Dienst - Leistung(s) - Arbeit: Kundenorientierung und Leistung in tertiären Organisationen, München, S. 99-118

Kern, H./Sabel, C. F. (1994): VerblaßteTugenden: Zur Krise des deutschen Produktionsmodells, in: Beckenbach, N./Van Treeck, W. (Hrsg.): Umbrüche gesellschaftlicher Arbeit, Soziale Welt, Sonderband 9, Göttingen, S. 605-624 Kotthoff, H. (1997): Führungskräfte im Wandel der Firmenkultur: Quasi-Unternehmer oder Arbeitgeber?, Berlin

Kratzer, N./Nies, S. (2009): Neue Leistungspolitik bei Angestellten: ERA, Leistungssteuerung, Leistungsentgelt, Berlin

Lane, C. (1989): Management and Labour in Europe:The Industrial Enterprise in Germany, Aldershot

von Behr, M. (2006): Industriearbeit im interkulturellen Kontext, in: Dunkel, W./ Sauer, D. (Hrsg.): Von der Allgegenwart der verschwindenden Arbeit: Neue Herausforderungen für die Arbeitsforschung, Berlin, S. 111-136

Volkswagen Aktiengesellschaft (2012): AutoUni-Programm, Frühjahr/Sommer 2012

\section{AUTOREN}

TABEA BROMBERG, Dr., Wissenschaftliche Mitarbeiterin in der Forschungs abteilung Arbeitszeit und Arbeitsorganisation am Institut Arbeit und Qualifikation (IAQ) der Universität Duisburg-Essen. Arbeitsschwerpunkte: Industrielle Beziehungen, Arbeits- und Produktionssysteme.

tabea.bromberg@uni-due.de

THOMAS HAIPETER, PD Dr., Leiter der Forschungsabteilung Arbeitszeit und Arbeitsorganisation am IAQ. Arbeitsschwerpunkte: Arbeits- und Industriesoziologie, Arbeitsbeziehungen und -regulierung.

thomas.haipeter@uni-due.de

ANGELIKA KÜMMERLING, Dr. phil., Wissenschaftliche Mitarbeiterin in der Forschungsabteilung Arbeitszeit und Arbeitsorganisation am IAQ. Arbeitsschwerpunkte: Arbeitszeit und Erwerbstätigkeit, Geschlechter- und internationale Vergleiche sowie die Entwicklungen in der Pflegebranche.

angelika.kuemmerling@uni-due.de 